\title{
Análisis teórico sobre la reestructuración de la cartera de negocios y su implicación empresarial
}

\author{
Angélica María Sánchez-Riofrío* \\ UNIVERSIDAD DE ESPECIALIDADES ESPÍRITU SANTO, ECUADOR
}

\author{
Daniel Molina-Ycaza** \\ EQ LAB, ECUADOR
}

\section{RESUMEN}

El objetivo principal de este trabajo es analizar conceptualmente el estudio de la reestructuración de la cartera de negocios $(\mathrm{RCN})$ y su efecto en los resultados de las empresas. Para el desarrollo del presente trabajo se adopta una metodología cualitativa mediante una revisión de diferentes fuentes literarias que incluyen tanto artículos científicos como libros. De acuerdo a la evidencia presentada, encontramos que los mecanismos más utilizados para la RCN son: (i) desinversiones (venta de activos, subsidiaria y empresa filial parcial), y (ii) fusiones y adquisiciones. Adicionalmente, se observa que de acuerdo a la literatura científica, estos mecanismos tienen, por lo general, un efecto positivo en los resultados de las empresas a excepción de las fusiones $\mathrm{y}$ adquisiciones que tienen un efecto en mayor parte negativo.

Palabras clave: Reestructuración de cartera; direcciones; mecanismos; desempeño; creación de valor

\begin{abstract}
The main objective of this work is to analyze conceptually the business portfolio restructuring (BPR) and its effect on company performance. For the development of the present work, the authors applied qualitative tools through a review of different literary sources that include both scientific articles and books. According to the evidence presented, we found that the most frequently used mechanisms for BPR are: (i) divestments (sell-off, spin-off and equity carve-out), and (ii) mergers and acquisitions. Additionally, these mechanisms have, in general, a positive effect on the results of the companies except for mergers and acquisitions that have an effect mostly negative.
\end{abstract}

Keywords: Portfolio restructuring; directions; mechanisms; performance; value creation

amsanche@uees.edu.ec*

demolina@uees.edu.ec** 


\section{INTRODUCCIÓN}

a reestructuración corporativa es una estrategia a nivel empresarial que comprende una gran variedad de acciones que tienen como objetivo la creación de valor para la empresa junto a un mejor rendimiento económico. Esta estrategia ha sido uno de los principales temas de investigación en el campo de la administración estratégica a lo largo de los años (Schonhaar, Nippa, \& Pidun, 2014). De acuerdo a la literatura científica, la reestructuración corporativa se divide en tres tipos de operaciones: reestructuración de la cartera de negocios $(\mathrm{RCN})$, reestructuración financiera y reestructuración organizativa. No obstante, en el desarrollo del presente trabajo se examinará a la RCN debido a lo complejo que resulta desarrollar un estudio integral de la reestructuración corporativa por su gran amplitud.

Debido al reto que tienen las empresas por competir en un mercado cada vez más exigente, se pone de manifiesto la necesidad de realizar un análisis sobre la estrategia de RCN y su efecto en los resultados empresariales. Ante tal situación, y con el fin de brindar otra opción a los administradores para solucionar problemas como financiamiento y destrucción de valor; el objetivo principal de este trabajo consiste en analizar conceptualmente el estudio de la RCN y su efecto en la creación de valor para las empresas.

En este trabajo se aplican herramientas cualitativas a través de una revisión de diferentes fuentes bibliográficas que incluyen tanto artículos científicos como libros relacionados al tema. Considerando otros artículos que realizan revisiones cualitativas (Ismail, Abdou, \& Annis, 2011; Sánchez-Riofrío, Guerras-Martín, \& Forcadell, 2016); los artículos revisados aquí han sido elegidos basándose en tres criterios: primero, que se encuentren en base de datos reconocidas a nivel mundial como Scopus e ISI Web of Science; segundo, por su actualidad; y tercero, por su relevancia dentro del tema estudiado medido a través del número de citas recibidas.

El presente artículo se estructura de la siguiente manera. El apartado II define los aspectos conceptuales para el estudio de la RCN. El apartado III presenta las direcciones de desarrollo para la RCN diferenciando al reenfoque, reposicionamiento y liquidación. El apartado IV plantea las clases de mecanismos más utilizados por 
la literatura para implementar la RCN. El apartado V muestra si el efecto obtenido sobre el resultado empresarial ha sido positivo, negativo o neutral de acuerdo a diversos estudios empíricos. Finalmente, el apartado VI contiene las conclusiones del artículo.

\section{Aspectos ReleVANTES DE LA RCN}

Desde la década de 1980, miles de empresas han utilizado la reestructuración corporativa para concentrarse en sus actividades clave y eliminar sinergias negativas (Bergh, Johnson, \& Dewitt, 2008; Hoskisson, Cannella, Tihanyi, \& Faraci, 2004; Markides, 1992). A través de este tipo de operaciones, los administradores pueden generar efectivo rápidamente a fin de reducir los niveles de deuda (David, 2013), descontinuar actividades con débil posición competitiva, o actividades en las que se obtienen bajos márgenes de rentabilidad (Fernández, Garay, \& Pablo, 2010).

De vez en cuando, la implementación de esta estrategia puede incitarse por circunstancias especiales, por ejemplo, cuando una empresa tiene la oportunidad de realizar una compra tan grande e importante que debe vender varias Unidades Estratégicas de Negocio (UEN) tanto para financiar la nueva adquisición (Thompson, Strickland, \& Gamble, 2012), como invertir en una expansión de las principales actividades que realiza la empresa, y así, obtener economías de escala. (Tarjizán, 2008).

La literatura científica sugiere múltiples formas de cambios en las empresas distinguiendo tres tipos de transacciones: (i) reestructuración de la cartera de negocios (desinversiones, fusiones y adquisiciones), (ii) reestructuración financiera (recapitalizaciones, compras apalancadas y cambios en la estructura de capital), y (iii) reestructuración organizativa (recorte de personal y rediseño de la división) (Gibbs, 1993; Ofek, 1993; Wood, 2001; Byerly, Lamontb, \& Keasler, 2003; Hoskisson, Johnson, Tihanyi, \& White, 2005; Schonhaar, Nippa, \& Pidun, 2014; Sánchez-Riofrío, GuerrasMartín, \& Forcadell, 2016). Estas tres clases de transacciones no se excluyen mutuamente, y de hecho, con frecuencia ocurren en conjunto (Ofek, 1993; Gibbs, 1993).

El origen de la RCN o también denominada reestructuración de activos, se deriva de tres raíces: economía, administración y finanzas 
(Sánchez-Riofrío, Guerras-Martín, \& Forcadell, 2015). En la tabla 1 se muestran diversos conceptos utilizados por la literatura científica para conceptualizar a profundidad la RCN.

\section{Tabla 1: Conceptos de reestructuración de la cartera de negocios}

\begin{tabular}{|l|c|}
\hline \multicolumn{1}{|c|}{ Concepto } & Referencia \\
\hline $\begin{array}{l}\text { La reestructuración de activos es una estrategia que implica modifi- } \\
\text { caciones en la composición de la cartera de negocios planteada como } \\
\text { respuesta a una situación de destrucción de valor. Se implementa a } \\
\text { través de diversos métodos de desarrollo que sirven para realizar ajustes } \\
\text { a los límites de la empresa con el objetivo de crear valor. }\end{array}$ & $\begin{array}{c}\text { Forcadell, \& } \\
\text { Guerras-Martín, } \\
\text { 2009) }\end{array}$ \\
\hline $\begin{array}{l}\text { La reestructuración de la cartera de negocios implica cambios significa- } \\
\text { tivos en el grupo de activos que la empresa posee o en líneas de negocio } \\
\text { que maneja una empresa, incluyendo liquidaciones, desinversiones, }\end{array}$ & $\begin{array}{c}\text { Singh, Useem, \& } \\
\text { Bhadury, 1999) }\end{array}$ \\
$\begin{array}{l}\text { ventas de activos y empresas filiales. } \\
\text { improestructuración de la cartera de negocios implica la venta de activos } \\
\text { supone adquisiciones que refuercen las actividades clave brindando } \\
\text { una fisonomía totalmente nueva a la composición de los negocios en } \\
\text { la empresa. }\end{array}$ & $\begin{array}{c}\text { (Dess, Lumpkin, } \\
\text { \& Eisner, 2011) }\end{array}$ \\
\hline $\begin{array}{l}\text { La reestructuración de activos realiza enajenaciones y adquisiciones a a } \\
\text { los negocios de una empresa, a través de la venta de activos, empresas } \\
\text { filiales, empresas filiales parciales, o fusiones y adquisiciones. }\end{array}$ & $\begin{array}{c}\text { (Renneboog \& } \\
\text { Szilagyi, 2006) }\end{array}$ \\
\hline $\begin{array}{l}\text { La reestructuración de la cartera de negocios se refiere a una estrategia } \\
\text { corporativa que afecta a las actividades de una empresa mediante las } \\
\text { desinversiones y adquisiciones. Se entiende como una salida alternativa } \\
\text { a una situación de destrucción de valor que muchas empresas enfrentar } \\
\text { por dificultades internas y externas. }\end{array}$ & $\begin{array}{c}\text { (Sánchez-Riofrío, } \\
\text { \& Forras-Martín, } \\
\text { 2017) }\end{array}$ \\
\hline $\begin{array}{l}\text { La reestructuración de activos se puede definir como un ajuste estra- } \\
\text { tégico intencional en la cartera de negocios de la empresa. Abarca la } \\
\text { reasignación de recursos para apoyar a algunos negocios sobre otros, } \\
\text { así como una serie de adquisiciones y desinversiones que buscan una } \\
\text { nueva, y más competitiva configuración de la empresa. }\end{array}$ & $\begin{array}{c}\text { Nippa, \& Pidun, } \\
\text { 2014) }\end{array}$ \\
\hline
\end{tabular}

Fuente: Elaboración propia.

A partir de los conceptos propuestos por distintos autores en la tabla anterior, se pueden recalcar un conjunto de situaciones específicas: primero, todas las definiciones indican que la estrategia se enfocará en la empresa mediante sus activos y líneas de negocios. Segundo, los conceptos seńalan distintos mecanismos para implementar los cambios correspondientes como desinversiones, fusiones y adquisiciones. Finalmente, la aplicación de la RCN 
pretende mejorar el desempeño organizacional mediante la creación de valor.

Luego de haber analizado las características principales de la $\mathrm{RCN}$, en este artículo se propone la siguiente definición: "La RCN es una modificación estratégica intencional en la composición de los negocios de la empresa mediante mecanismos como desinversiones, fusiones y adquisiciones con el objetivo de crear valor para los accionistas".

\section{Direcciones de DesarRollo EN LA RCN}

El estudio de la RCN a lo largo de los años se ha examinado bajo diversos enfoques, sin embargo, entre ellos sobresalen: la teoría de la agencia (Jensen, 1986), los costos de transacción (Williamson, 1975) y el enfoque basado en recursos (EBR) (Penrose, 1959). En el ámbito empresarial, tanto la teoría de la agencia como los costos de transacción han sido utilizados principalmente para el estudio del gobierno corporativo, desinversiones y exceso de diversificación; mientras que se ha recurrido al EBR para explicar el reenfoque corporativo, la organización de la cartera basada en los recursos y las capacidades esenciales disponibles de la empresa (Sánchez-Riofrío, Guerras-Martín, \& Forcadell, 2015). Para efectos de profundizar en un mayor análisis, este estudio se concentra en el EBR.

Desde una perspectiva de direccionamiento, la RCN puede dividirse en: reorientación corporativa, reposicionamiento corporativo y liquidación de activos (Markides, 1992; Gibbs, 1993; Byerly, Lamontb, \& Keasler, 2003; Hoskisson, Cannella, Tihanyi, \& Faraci, 2004; Choe \& Roehl, 2007; Bergh, Johnson, \& Dewitt, 2008; Schonhaar, Nippa, \& Pidun, 2014).

\subsection{REORIENTACIÓN CORPORATIVA (CORPORATE REFOCUSING)}

Durante 1980, los administradores sintieron una creciente presión de reenfocar sus operaciones para maximizar el valor de sus empresas (Bhana, 2006). La reorientación corporativa tal vez es la forma más común de RCN en años recientes implicando una contracción del volumen de operaciones junto a la concentración del negocio principal (Byerly, Lamontb, \& Keasler, 2003; Markides, 1992). Es decir, al emplear esta dirección, una empresa puede reducir el número 
de negocios mediante su venta (Hoskisson, Johnson, Tihanyi, \& White, 2005) o división en distintas partes (Tarjizán, 2008) a fin de eliminar actividades periféricas (Lockett \& Thompson, 2001) y concentrarse en sus actividades clave caracterizadas por tener perspectivas de mayores márgenes de ganancias (Robbins \& Pearce, 1992).

Colak (2010) y Schonhaar, Nippa, \& Pidun (2014) establecen a la diversificación como una dirección en la RCN, no obstante, en la literatura académica, sobre todo de Management, (Dess, Lumpkin, \& Eisner, 2011; Thompson, Strickland, \& Gamble, 2012; Hitt, Ireland, \& Hoskisson, 2015) se la evidencia como otra estrategia a nivel corporativo. A diferencia de los autores anteriormente mencionados, en este trabajo no se considera a la diversificación como otra clase de dirección; sin embargo, se observa una relación directa con la RCN ya que la empresa debe encontrarse obligatoriamente diversificada para poder especializarse en su actividad clave.

Las empresas excesivamente diversificadas, por lo general, presentan negocios muy dispersos con estrategias que resultan difíciles de manejar eficientemente. Por esto, dichas empresas tienden a tomar en consideración los beneficios que posee una estrategia de reorientación. Para Gibbs (1993), la reducción de la diversificación en la cartera está relacionada con oportunidades de inversión y creación de valor. De forma similar, John \& Ofek (1995) aseguran que las empresas demasiado diversificadas luego de la reorientación obtienen un aumento en el desempeño, y un retorno significativamente positivo.

Con motivo de analizar esta dirección en el ámbito empresarial, se toma como ejemplo a la compañía Obrascón Huarte Lane (OHL). A inicios del 2018, esta empresa aprobó la venta de OHL Concesiones al fondo australiano IFM acordado por 2.235 millones de euros. Esta desinversión se realiza siguiendo un plan de reorientación corporativa para el saneamiento y reestructuración de la empresa a fin de reducir su deuda y volver a la rentabilidad deseada ("El Economista", Madrid, Enero 08 del 2018). 
3.2 REPOSICIONAMIENTO CORPORATIVO (CORPORATE REPOSITIONING) La dirección de reposicionamiento desarrollada por Byerly, Lamontb, \& Keasler (2003) se encarga de redefinir la actividad principal y reconfigurar los activos alrededor de un núcleo recién definido. Las operaciones para su implementación pueden incluir una serie de desinversiones seguido de adquisiciones que refuercen las nuevas actividades clave. Es decir, en el transcurso del reposicionamiento de la cartera, algunos negocios de la empresa pueden ser vendidos, nuevas unidades pueden ser adquiridas y los recursos de los antiguos negocios principales serán redirigidos hacia un nuevo núcleo designado. Schonhaar, Nippa, \& Pidun (2014) señalan que esta dirección es la forma más extrema de RCN debido a que la empresa abandona completamente su estrategia corporativa y opera en un segmento de negocio totalmente distinto.

En el mundo existe un sinnúmero de empresas que han utilizado el reposicionamiento corporativo tales como Nokia, Colgate, Peugeot, entre otras. Para contextualizar esta estrategia, un ejemplo concreto sería lo sucedido con Samsung. En 1938, Byung-Chull Lee, inició esta empresa centrada en la venta de pescado seco, frutas y verduras. A través del tiempo, mediante diversas adquisiciones y desinversiones, Samsung reestructuró su cartera de negocios incursionando por diversos sectores como la industria pesada, química y petroquímica hasta finalmente convertirse en una de las corporaciones más importantes del mundo en cuanto a tecnología (Samsung, 2005).

\subsection{LiQuidación de ACTIVOS}

La liquidación de activos es la decisión más importante que se puede tomar a nivel de empresa. Esta dirección consiste en concluir las operaciones del negocio y por consiguiente iniciar un proceso de desinversiones que viene encaminado a pagar la totalidad de los pasivos. De acuerdo a Kim \& Schatzberg (1987) las ganancias obtenidas por las liquidaciones son utilizadas para pagar la deuda existente y los fondos restantes son distribuidos a los accionistas como dividendos. En caso de que la empresa no encuentre un comprador dispuesto a pagar un valor aceptable en el momento de la decisión, se determinará si debe mantener el negocio hasta que encuentre un 
comprador, o en el caso de que el negocio esté agobiado por crisis y pierda mucho dinero, simplemente deberá cerrarlo y liquidar sus activos restantes (Thompson, Strickland, \& Gamble, 2008).

Gallardo (2012) manifiesta que la liquidación es aplicable cuando la empresa ha seguido una dirección de reenfoque y no ha tenido éxito; cuando la única alternativa de la empresa es la quiebra; o cuando los accionistas pueden disminuir sus pérdidas vendiendo los activos de la organización.

En muchas ocasiones, la falta de factores esenciales como la innovación, el capital humano especializado y la financiación (Molina-Ycaza \& Sánchez-Riofrío, 2016) conllevan al cierre de las operaciones en muchas empresas. Un ejemplo conocido a nivel mundial es lo sucedido con la empresa Blockbuster. Ante la exigente competencia que planteó Netflix llevando su mercado a la plataforma online, Blockbuster no tuvo la capacidad suficiente de innovar para competir en un entorno cada vez más digitalizado. A los pocos ańos, gradualmente tuvo que cerrar sus locales hasta el punto de haber liquidado todos los activos a fin de que sus accionistas no sufran mayores pérdidas a las obtenidas hasta ese momento (IngaHenríquez \& Caba-Gajardo, 2015). En el gráfico 1 se resumen las tres direcciones correspondientes a la RCN. 


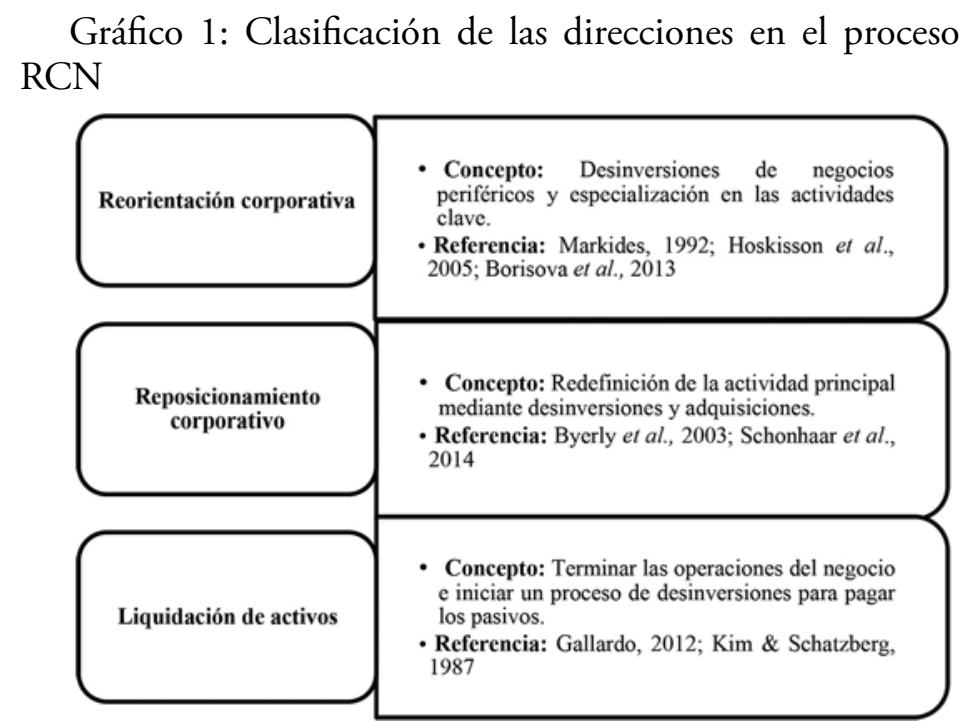

Fuente: Elaboración propia.

\section{MeCANismos PARA IMPLEMENTAR LA RCN}

La literatura científica muestra una diversidad de opciones por las cuales una empresa puede entrar en el proceso de implementación de la RCN. En este apartado se propone una segmentación (ver tabla 2) para clasificar los estudios existentes en base a los mecanismos más utilizados en la RCN.

\subsection{Desinversiones}

La desinversión consiste en eliminar activos, líneas de negocios e inversiones por medio de su venta (Fernández, Garay, \& Pablo, 2010; Gallardo, 2012; Duhaime \& Grant, 1984) o división a una empresa adquiriente que toma el control. Durante los periodos de recesión, las desinversiones representan un canal de recaudación de fondos alternativos para las empresas que no tienen acceso a los mercados de capital (Borisova, John, \& Salotti, 2013; Lang, Poulsen, \& Stulz, 1995).

Abdul-Magid \& Hardy (2004) y Fernández, Garay \& Pablo (2010) han identificado en los estudios financieros tres motivos para una desinversión. En primer lugar, los activos pueden ser transferidos 
a quienes consiguen operarlo de forma más productiva, es decir, la compañía vendedora debe buscar en qué empresa representaría el negocio una buena correspondencia y en qué condiciones lo vería como un buen trato. En segundo lugar, las desinversiones reducen el grado de diversificación y permiten la concentración en sus actividades clave. En tercer lugar, las desinversiones aumentan los ingresos de la empresa por lo que disminuye la necesidad de tener que recurrir al mercado de capitales para adquirir financiamiento.

Uno de los argumentos más populares para la RCN es que los administradores utilizan desinversiones en líneas de negocios no relacionadas con motivo de reducir empresas excesivamente diversificadas a un punto en el que se pueda gestionar de forma más económica y competitiva (Gibbs, 1993; Bergh, Johnson, \& Dewitt, 2008). Al retirarse de estas líneas de negocios, la empresa puede racionalizar su organización y coordinación de las actividades comerciales entre sus unidades de negocios más adecuadas. Esto permitiría concentrar sus recursos y esfuerzos en el fortalecimiento de sus competencias básicas para el largo plazo (Choe \& Roehl, 2007).

La literatura existente señala que las desinversiones pueden tomar varias formas, entre las más utilizadas se encuentran: venta de activos (sell-off), empresa filial (spin-off), y empresa filial parcial (equity carve-out) (Slovin, Sushka, \& Ferraro, 1995; Abdul-Magid \& Hardy, 2004; Hoskisson, Johnson, Tihanyi, \& White, 2005; Renneboog \& Szilagyi, 2006; Tarjizán, 2008; Bergh, Johnson, \& Dewitt, 2008; Uddin, 2010; Damaraju, Barney, \& Makhija, 2011).

\subsubsection{Venta de aCtivos (SELl-OFf)}

Vender directamente un negocio a otra compañía es por mucho, la opción más utilizada de desinversión (Thompson, Strickland, \& Gamble, 2008). La venta de activos representa un tipo de financiamiento y reestructuración corporativa que se extiende cada vez más allá de los mercados nacionales para involucrar a compradores extranjeros (Borisova, John, \& Salotti, 2013). Como se observa en el gráfico 2, esta operación es considerada como la transferencia voluntaria de las partes de una empresa (empresa "A" vendedora), ya sean instalaciones, equipos, $\mathrm{y}$ divisiones a terceros 
(empresa "B" compradora) (Fernández, Garay, \& Pablo, 2010; Abdul-Magid \& Hardy, 2004) por lo general, a cambio de efectivo (Tarjizán, 2008; Bhana, 2006).

Gráfico 2: Representación de la venta de activos

Antes:

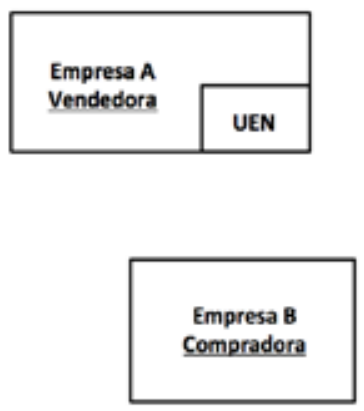

Después:

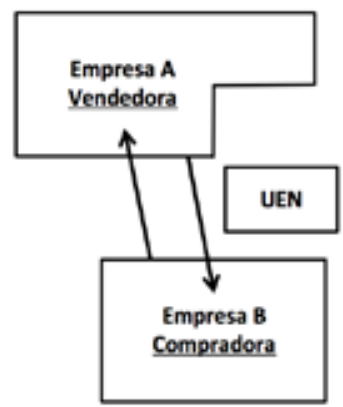

Fuente: Elaboración propia.

Durante situaciones en que las empresas tienen la necesidad de obtener dinero en efectivo, los administradores pueden recurrir a la venta de activos para liquidar sus deudas emitidas en dificultades financieras (Shleifer \& Vishny, 1992). Lang, Poulsen, \& Stulz (1995) señalan que generalmente las empresas que venden sus activos tienen un pobre desempeńo y/o un apalancamiento significativo. Sin embargo, existen diversos casos donde las empresas que gozan de condiciones financieras sanas, venden sus activos puesto que necesitan liquidez para realizar una compra o para poder invertir en proyectos de valor, y en otras UEN proyectadas con mejores oportunidades de crecimiento (Fernández, Garay, \& Pablo, 2010; Sibilkov, Straska, \& Waller, 2013).

John \& Ofek (1995) sostienen que la buena disposición del comprador a pagar demasiado es una de las principales razones para la venta de activos. En este sentido, las empresas con grandes flujos de caja libre (free cash flows) pueden adquirir una división a un precio superior de su valor económico real (Jensen, 1986). En cuanto a los beneficios que se obtienen por este mecanismo, Cheng, Aerts, \& Jorissen (2010) señalan que la venta de activos conduce a una mejora significativa en el rendimiento operativo a largo plazo. Los 
recursos generados por la venta de activos se utilizan habitualmente para pagar deuda (Shleifer \& Vishny, 1992). Además, pueden ser entregados directamente a los accionistas a prorrata del número de acciones que posean en la empresa vendedora a través de dividendos (Tarjizán, 2008).

Para situar este mecanismo en el contexto empresarial se puede mencionar lo sucedido a inicios del 2017, cuando Petróleo Brasileño S.A. (Petrobras) anunció la venta de sus activos en Chile al fondo de inversiones Southern Cross Group por US\$470 millones. La venta señalada se enmarca en el proceso de desinversiones lanzado por la petrolera para recaudar dinero a fin de hacer frente a la gigantesca deuda que arrastra de varios años atrás (“América Economía”, Río de Janeiro, Enero 05 del 2017).

\subsubsection{Empresa Filial o SUbSidiaria (SPIN-OFF)}

Cuando una UEN posee múltiples recursos para poder salir al mercado a competir por sí misma, la empresa matriz (tal como se observa el gráfico 3) toma la decisión de separarla como una empresa independiente distribuyendo las acciones a los actuales accionistas de la empresa (Thompson, Strickland, \& Gamble, 2008; Fernández, Garay, \& Pablo, 2010; Tarjizán, 2008; Renneboog \& Szilagyi, 2006; Wallin, 2012; Uddin, 2010; Bhana, 2004; Seward \& Walsh, 1996). La empresa filial es una manera por la cual una empresa puede desinvertir una división y mejorar su concentración (Bhana, 2004; Uddin, 2010). Los administradores utilizan este mecanismo para reestructurar parte de la empresa en unidades independientes que proporcionan una mayor claridad acerca del potencial de los activos y el potencial de sus ganancias (Bergh, Johnson, \& Dewitt, 2008). 
Gráfico 3: Representación de la empresa filial

Antes:

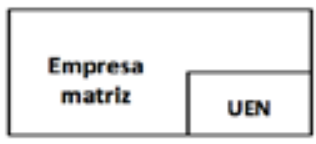

Después:

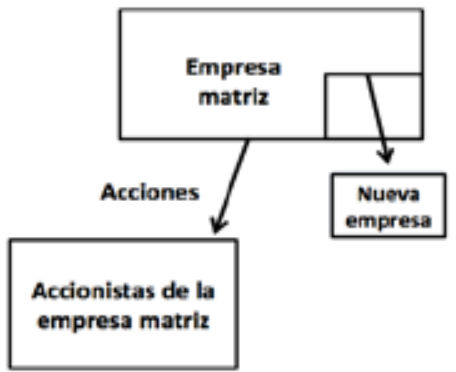

Fuente: Elaboración propia.

Entre los beneficios de este mecanismo, Uddin (2010) sostiene que la subsidiaria puede crear valor debido a: la reducción de las deseconomías de funcionamiento e ineficiencia, la reducción de la asimetría de información, la reducción de la deuda tributaria, y la mejora del gobierno corporativo en la empresa matriz reestructurada y su filial. Asimismo, Daley, Mehrotra, \& Sivakumar (1997) afirman que la subsidiaria crea valor cuando incrementa su concentración corporativa mediante las mejoras en el rendimiento tras la operación.

Una de las características principales de la empresa filial es que se constituye como un único modo de desinversión que no involucra transacciones en efectivo ya que esta no es motivada por el deseo o la necesidad de generar efectivo a fin de pagar deuda como sucede con otras formas de desinversión (Bhana, 2004; Bergh, Johnson, \& Dewitt, 2008; Renneboog \& Szilagyi, 2006; Michaely \& Shaw, 1995; Daley, Mehrotra, \& Sivakumar, 1997).

Contextualizando este mecanismo a nivel empresarial, se pone de manifiesto el ejemplo de Google. Desde el 2015, esta compañía cambió su estructura empresarial pasando a llamarse Alphabet. A su vez, independizó sus unidades estratégicas de negocio dividiéndose en Google, Calico, Nest, Fiber, Xlab, Venture y Lab. La empresa decidió que había llegado la hora de recuperar el espíritu emprendedor. Por eso, se dividió en siete compañías más pequeñas y separó su fuente de ingresos seguros de las ideas incipientes debido a 
que quería aligerar el paso de todas las filiales, y, además, contener el posible dańo de proyectos arriesgados ("El País”, Las Vegas, Agosto 11 del 2015).

\subsubsection{EMPresa filial PARCIAL (EQUity CARVE-OUT)}

La empresa filial parcial (EFP), tal como se observa en el gráfico 4, es una forma de reestructuración que se produce cuando la empresa matriz vende una participación minoritaria (por lo general 20\% o más) que posee en una subsidiaria a los inversionistas mediante la oferta pública inicial (OPI) (Fernández, Garay, \& Pablo, 2010; Thompson, Strickland, \& Gamble, 2008; Desai, Klock, \& Mansi, 2011; Hulburt, 2011; Perotti \& Rossetto, 2007; Powers, 2003; Slovin, Sushka, \& Ferraro, 1995).

Gráfico 4: Representación de la empresa filial parcial

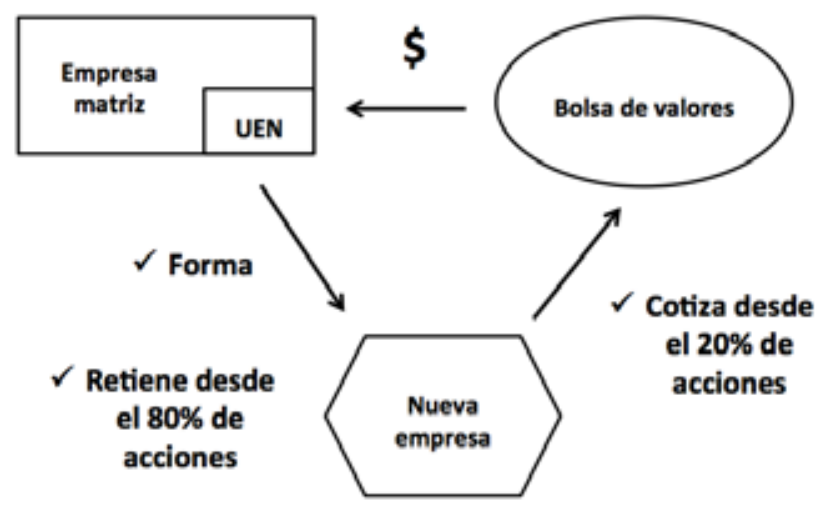

Fuente: Elaboración propia.

La EFP difiere sustancialmente de otras formas de reestructuración (Desai, Klock, \& Mansi, 2011). Sin embargo, este mecanismo es similar a la subsidiaria, en términos, de que ambos son formas de desinversiones corporativas (Hulburt, 2011). Michaely \& Shaw (1995) argumentan la existencia de tres diferencias principales entre la subsidiaria y la EFP. En primer lugar, las acciones de una subsidiaria se distribuyen a los accionistas existentes, por el contrario, 
la EFP establece un nuevo conjunto de accionistas. En segundo lugar, las acciones emitidas a través de la EFP generan flujo de caja positivo para la empresa, mientras que una subsidiaria no tiene consecuencias de inmediata liquidez. Finalmente, las empresas que se desprenden mediante la EFP incurren en gastos de desembolso significativamente mayores.

Schipper \& Smith (1986) describen tres razones importantes para establecer una EFP: financiación, reestructuración y liberación de valor. La razón de financiación consiste en las instancias cuando la matriz realiza una EFP para financiarse a sí misma o a proyectos de crecimiento de su subsidiaria, además, sirve para aliviar la deuda de la matriz o recaudar fondos que le permitan cubrir las necesidades del gasto de capital. La razón de la reestructuración radica en los casos cuando la matriz recorta la filial para obtener ganancias y, finalmente, la liberación de valor se fundamenta en situaciones cuando la matriz compromete una EFP para que los inversores entiendan mejor las operaciones de la división. Este último argumento es ratificado por Desai, Klock, \& Mansi (2011) al manifestar que la motivación de la matriz para llevar a cabo este tipo de operaciones se produce con motivo de desbloquear el valor de la filial.

En décadas pasadas las teorías existentes generalmente consideraban a la venta de activos como la única fuente de financiación sin tomar en cuenta a la EFP (Shleifer \& Vishny, 1992). Actualmente, las empresas que desean financiar oportunidades de crecimiento a futuro o aumentar la distribución de efectivo a los accionistas actuales prefieren la ruta de la EFP dado que la empresa matriz recibe dinero por las acciones vendidas al público en general. Además, es necesario que la empresa matriz tenga un nivel del 50\% al $80 \%$ de la propiedad en una filial si desea mantener el control y sus estados financieros consolidados (Michaely \& Shaw, 1995). No obstante, luego de que las empresas utilicen este mecanismo, las mismas pueden volver a vender otra parte de sus acciones mediante una oferta pública secundaria. Cabe resaltar que cuando la empresa matriz retiene menos del $50 \%$ de las acciones, usualmente suelen desinvertir completamente la filial.

En 1999, Siemens constituyó una EFP la cual denominó Infineon Techonologies AG especializada en la industria de semiconductores. 
Siemens mantuvo el $71 \%$ de las acciones de Infineon vendiendo el $29 \%$ restante en la bolsa de valores mediante la OPI. Para el 2001, redujo el control de la empresa a un 50,9\% con el fin de recaudar más fondos para realizar diversas actividades estratégicas (McKinsey \& Company, New York, Septiembre del 2001).

\subsection{FuSIONES Y ADQUISICIONES}

Las fusiones y adquisiciones (F\&A) implican la unión de dos o más empresas (Allen, Lummer, McConell, \& Reed, 1995) independientemente del sector en el que se encuentre para formar una nueva entidad que permita ingresar a nuevos mercados o a una nueva área de negocio (Malik, Anuar, Khan, \& Khan, 2014). Las adquisiciones se utilizan con frecuencia cuando la empresa carece de competencias distintivas que le permita competir en una industria, por ende, dispone de su capital para comprar una empresa establecida que sí las posea (Hill \& Jones, 2009; Malik, Anuar, Khan, \& Khan, 2014).

En las tres última décadas, un sinnúmero de empresas han utilizado las F\&A como herramienta de reestructuración corporativa (Malik, Anuar, Khan, \& Khan, 2014). Por lo general, se ajustan bien cuando las alianzas y asociaciones no alcanzan a dar a la empresa acceso a los recursos y capacidades deseadas (David, 2013), y en diversos casos, se presenta como la mejor opción para salir adelante ampliando los límites de propiedad (Ismail, Abdou, \& Annis, 2011).

Los motivos potenciales para las F\&A se discuten ampliamente en la literatura académica. Mantravadi \& Reddy (2008) afirman que entre los objetivos detrás de este mecanismo se encuentran: la mejora de ingresos y rentabilidad, la adquisición de nuevas tecnologías, y tiempo más rápido en el mercado. Por su lado, Sulaiman (2012) señala que se utilizan con motivo de mejorar la competitividad de las empresas y obtener ventajas competitivas. Esto se logra mediante una mayor participación de mercado, ampliando la cartera a fin de reducir riesgos potenciales y capitalizando economías de escala. En promedio, las adquisiciones aumentan el valor de las acciones combinadas de la empresa objetivo y la empresa adquirida, lo que sugiere que las adquisiciones crean valor para los accionistas (Yook, 2004). 
Renneboog \& Szilagyi (2006), Bena \& Li (2012) y Malik, Anuar, Khan, \& Khan (2014) dan énfasis a la existencia de aumentos sinérgicos que elevarían el valor de la empresa combinada. De acuerdo a Homberg, Rost, \& Osterloh (2007) existen diferentes fuentes de sinergias como las operativas que pueden derivarse de economías de escala o mayor poder de mercado. Asimismo, hay alcance de sinergias financieras, tales como menor coste de capital, deuda tributaria reducida o mejor eficiencia de capitales internos.

En contraste con estos autores, existe una considerable discrepancia en la literatura puesto que en gran parte de ella consideran que las F\&A no siempre tienen el éxito que las partes interesadas desean que tengan, muchas de ellas no añaden valor a la empresa adquiriente y, de hecho, a menudo acaban por disiparlo. Ismail, Abdou, \& Annis (2011) aseveran que las F\&A en la teoría son geniales, pero en la práctica, las cosas pueden ir mal. King, Dalton, Daily, \& Covin (2004) sostienen que las F\&A, en promedio, no contribuyen positivamente a la rentabilidad de una empresa compradora. Además, manifiestan que no es el mejor medio por el cual acceder a recursos valiosos existentes en otros negocios.

De la misma manera, Yook (2004) y Hill \& Jones (2009) indican que la razón más importante por la cual fracasan muchas adquisiciones es que la mejora del rendimiento operativo es aniquilada por los costos de capital de las grandes primas pagadas a la empresa objetivo, sin crear algún beneficio económico real para los accionistas de las empresas adquirentes. Finalmente, la teoría de flujo de caja libre de Jensen (1986) predice que las F\&A son más propensas a destruir, en vez de crear valor.

Para una mejor comprensión Thompson, Strickland, \& Gamble (2012) detallan otros motivos por los cuales las F\&A en ocasiones no logran obtener los resultados previstos. Entre los motivos más comunes se encuentran: el largo tiempo en que las capacidades competitivas pueden concretarse; las acciones para fusionar las culturas corporativas se detienen por la resistencia de los miembros de la empresa; y puede ser difícil resolver diferencias entre estilos de los administradores y procesos operativos. Adicionalmente, David (2013) resalta situaciones como la evaluación inadecuada del objetivo, grandes deudas, incapacidad de lograr sinergias, demasiada 
diversificación, y la baja moral de los empleados debido a despidos y reubicaciones. Tales dificultades posteriores a la integración pueden mantener las unidades adquiridas menos integradas en el sistema de negocios del grupo de adquisición, aumentando la probabilidad de que luego sean revendidas (Choe \& Roehl, 2007).

En 2016, se realizó una de las fusiones más importantes de la historia cuando $\mathrm{AB}$ InBev, la empresa cervecera más grande del mundo, adquirió a SAB Miller por más de US\$100,000 millones. Dicha fusión se constituyó por diversos motivos tales como: obtener sinergias positivas, consolidarse como la empresa cervecera más grande del mundo prácticamente sin contendientes ya que $\mathrm{SAB}$ Miller era su competencia directa, aprovechar el mercado de África en el que SAB Miller se encuentra mejor posicionado y fortalecer la posición en Asia (The Wall Street Journal, New York, Septiembre 16 del 2015).

Con motivo de conceptualizar todo lo expuesto a lo largo de este apartado, en la tabla 2 se sintetizan los conceptos de los mecanismos más utilizados en la RCN.

Tabla 2: Clasificación de los mecanismos más utilizados en la RCN

\begin{tabular}{|c|c|c|}
\hline Mecanismos & Concepto & Referencia \\
\hline Desinversión & $\begin{array}{l}\text { La desinversión consiste en eliminar acti- } \\
\text { vos, líneas de negocios, o inversiones por } \\
\text { medio de su venta a una empresa adqui- } \\
\text { riente que toma el control. Este mecanis- } \\
\text { mo se divide en: venta de activos, empresa } \\
\text { filial o subsidiaria, y empresa filia parcial. }\end{array}$ & $\begin{array}{l}\text { Fernández, Garay, \& Pa- } \\
\text { blo, 2010; Gallardo, 2012; } \\
\text { Duhaime \& Grant, } 1984\end{array}$ \\
\hline $\begin{array}{l}\text { Venta de } \\
\text { activos } \\
\text { (sell-off) }\end{array}$ & $\begin{array}{l}\text { La venta de activos es la transferencia vo- } \\
\text { luntaria de las partes de una empresa, ya } \\
\text { sean instalaciones, equipos, y divisiones a } \\
\text { terceros, por lo general no relacionadas a } \\
\text { las actividades clave que realiza la empresa. }\end{array}$ & $\begin{array}{l}\text { Abdul-Magid \& Hardy, } \\
\text { 2004; Tarjizán, 2008; } \\
\text { Bhana, 2006; Fernández, } \\
\text { Garay, \& Pablo, } 2010\end{array}$ \\
\hline $\begin{array}{l}\text { Empresa filial } \\
\text { o Subsidiaria } \\
\text { (spin-off) }\end{array}$ & $\begin{array}{l}\text { La empresa matriz toma la decisión de } \\
\text { separar una división como empresa inde- } \\
\text { pendiente distribuyendo las acciones de la } \\
\text { filial a los actuales accionistas. }\end{array}$ & $\begin{array}{l}\text { Wallin, 2012; Uddin, } \\
\text { 2010; Bhana, 2004; } \\
\text { Seward \& } \\
\text { Walsh, 1996; Thompson, } \\
\text { Strickland, \& Gamble, } \\
\text { 2008; Renneboog \& Szi- } \\
\text { lagyi, 2006 }\end{array}$ \\
\hline
\end{tabular}




\begin{tabular}{|c|l|c|}
\hline $\begin{array}{c}\text { Empresa filial } \\
\text { parcial (Equi- } \\
\text { ty Carve-out) }\end{array}$ & $\begin{array}{l}\text { La empresa matriz vende una participa- } \\
\text { ción minoritaria que posee en su empresa } \\
\text { filial a los inversionistas mediante la oferta } \\
\text { pública inicial. }\end{array}$ & $\begin{array}{c}\text { Desai, Klock, \& Mansi, } \\
\text { 2011; Thompson, Strick- } \\
\text { land, \& Gamble, 2008; } \\
\text { Fernández, Garay, \& } \\
\text { Pablo, 2010; Perotti \& } \\
\text { Rossetto, 2007; Powers, } \\
\text { 2003; Slovin, Sushka, \& } \\
\text { Ferraro, 1995 }\end{array}$ \\
\hline $\begin{array}{c}\text { Fusiones y } \\
\text { adquisicio- } \\
\text { nes (F\&A) }\end{array}$ & $\begin{array}{l}\text { Las fusiones y adquisiciones implican la la de dos o más empresas para formar } \\
\text { unión dentidad que permite ingresar a nuevos } \\
\text { una entian, Anuar, Khan, \& } \\
\text { mercados potenciales o a una nueva área } \\
\text { de negocio. }\end{array}$ & $\begin{array}{c}\text { Malik, 2014; Allen, Lum- } \\
\text { mer, McConell, \& Reed, } \\
1995\end{array}$ \\
\hline
\end{tabular}

Fuente: Elaboración propia.

\section{El efecto de la estrategia de RCN SObre El REsultado} EMPRESARIAL

El efecto que posee la RCN en la creación de valor de las empresas es un tema de amplio interés en el ámbito académico. De la misma manera, un aspecto elemental para la administración estratégica es comprender cómo afecta esta estrategia en los resultados de las empresas. El objetivo de la reestructuración corporativa independientemente de la división que se estudie está orientado a la creación de valor (Markides, 1992; Sánchez-Riofrío, GuerrasMartín, \& Forcadell, 2015), es por esto que en la tabla 3 se muestra el efecto obtenido por la RCN en las empresas de acuerdo a distintos estudios empíricos.

El criterio para la elaboración de la siguiente tabla se basa en los diversos mecanismos propuestos en este trabajo (ver tabla 2). Cada uno de los estudios expuestos tiene un efecto que puede ser positivo, negativo y neutral para la creación de valor. Cabe señalar que cuando se determina que un efecto es neutral quiere decir que en la investigación de algún autor se consideran otras variables de las que dependen los resultados y por ellas no se establece un efecto determinado. Además, al considerarse que el efecto es negativo, no quiere decir que, en sí, sea completamente perjudicial para la empresa puesto que el enfoque principal es la creación de valor (generación de utilidades). Por lo cual, esto significa que si el resultado es negativo, el mecanismo implementado no ha creado valor para las empresas 


\section{en el estudio correspondiente.}

Tabla 3: El efecto post-reestructuración de las empresas en los resultados basado en estudios empíricos

\begin{tabular}{|c|c|c|c|c|c|}
\hline Mecanismo & Muestra & Región & Periodo & $\begin{array}{c}\text { Creación de } \\
\text { valor }\end{array}$ & Referencia \\
\hline $\begin{array}{c}\text { Fusiones y } \\
\text { adquisiciones }\end{array}$ & $\begin{array}{c}937 \\
\text { adquisiciones } \\
\text { y } 227 \text { ofertas } \\
\text { públicas }\end{array}$ & Estados Unidos & $\begin{array}{l}1955- \\
1987\end{array}$ & Negativo & $\begin{array}{l}\text { Agrawal, } \\
\text { Jaffe, \& } \\
\text { Mandelker } \\
\text { (1992) }\end{array}$ \\
\hline $\begin{array}{c}\text { Venta de } \\
\text { activos y } \\
\text { empresa filial }\end{array}$ & 205 empresas & Estados Unidos & $\begin{array}{l}1990- \\
1997\end{array}$ & Positivo & $\begin{array}{c}\text { Bergh \& } \\
\operatorname{Lim}(2008)\end{array}$ \\
\hline $\begin{array}{c}\text { Empresa filial } \\
\text { parcial }\end{array}$ & 189 eventos & Estados Unidos & $\begin{array}{l}1985- \\
1995\end{array}$ & Negativo & $\begin{array}{c}\text { Boone, } \\
\text { Haushalter, } \\
\& \\
\text { Mikkelson } \\
(2003)\end{array}$ \\
\hline $\begin{array}{l}\text { Venta de } \\
\text { activos }\end{array}$ & 1458 eventos & Estados Unidos & $\begin{array}{l}1998- \\
2008\end{array}$ & Positivo & $\begin{array}{l}\text { Borisova, } \\
\text { John, \& } \\
\text { Salotti } \\
(2013)\end{array}$ \\
\hline $\begin{array}{l}\text { Fusiones y } \\
\text { adquisiciones }\end{array}$ & 39 industrias & $\begin{array}{l}41 \text { países } \\
\text { alrededor del } \\
\text { mundo }\end{array}$ & $\begin{array}{l}1990- \\
2001\end{array}$ & Positivo & $\begin{array}{c}\text { Bris, } \\
\text { Brisley, \& } \\
\text { Cabolis } \\
(2008)\end{array}$ \\
\hline Empresa filial & 85 empresas & Varias regiones & $\begin{array}{l}1975- \\
1994\end{array}$ & Positivo & $\begin{array}{c}\text { Daley, } \\
\text { Mehrotra, } \\
\& \\
\text { Sivakumar } \\
(1997)\end{array}$ \\
\hline $\begin{array}{c}\text { Empresa filial } \\
\text { parcial }\end{array}$ & $\begin{array}{l}222 \text { eventos de } \\
199 \text { empresas }\end{array}$ & Varias regiones & $\begin{array}{l}1986- \\
2004\end{array}$ & $\begin{array}{c}\text { Neutral: } \\
\text { depende } \\
\text { de las } \\
\text { circunstancias }\end{array}$ & $\begin{array}{l}\text { Desai, } \\
\text { Klock, } \\
\text { \& Mansi } \\
(2011)\end{array}$ \\
\hline Empresa filial & 155 eventos & Varias regiones & $\begin{array}{l}1975- \\
1991\end{array}$ & Positivo & $\begin{array}{c}\text { Desai \& } \\
\text { Jain (1999) }\end{array}$ \\
\hline $\begin{array}{l}\text { Venta de } \\
\text { activos }\end{array}$ & 40 empresas & Varias regiones & $\begin{array}{l}1975- \\
1980\end{array}$ & Positivo & $\begin{array}{l}\text { Duhaime } \\
\text { \& Grant } \\
(1984)\end{array}$ \\
\hline
\end{tabular}




\begin{tabular}{|c|c|c|c|c|c|}
\hline $\begin{array}{l}\text { Venta de } \\
\text { activos }\end{array}$ & 107 eventos & $\begin{array}{l}\text { Latinoamérica, } \\
\text { España, } \\
\text { Estados } \\
\text { Unidos, y } \\
\text { Trinidad \& } \\
\text { Tobago }\end{array}$ & $\begin{array}{c}2000- \\
2006\end{array}$ & Positivo & $\begin{array}{c}\text { Fernández, } \\
\text { Garay, } \\
\text { \& Pablo } \\
(2010)\end{array}$ \\
\hline $\begin{array}{l}\text { Venta de } \\
\text { activos }\end{array}$ & 132 empresas & Reino Unido & $\begin{array}{l}1985- \\
1993\end{array}$ & Positivo & $\begin{array}{l}\text { Haynes, } \\
\text { Thompson, } \\
\text { \& Wright } \\
(2002)\end{array}$ \\
\hline $\begin{array}{l}\text { Venta de } \\
\text { activos }\end{array}$ & 413 eventos & Reino Unido & $\begin{array}{l}1993- \\
2000\end{array}$ & Negativo & $\begin{array}{l}\text { Hillier, } \\
\text { McColgan } \\
\text { (2009) }\end{array}$ \\
\hline Empresa filial & $\begin{array}{l}123 \text { eventos de } \\
166 \text { empresas }\end{array}$ & Varias regiones & $\begin{array}{l}1963- \\
1981\end{array}$ & Positivo & $\begin{array}{l}\text { Hite \& } \\
\text { Owers } \\
(1983)\end{array}$ \\
\hline $\begin{array}{c}\text { Empresa filial } \\
\text { parcial }\end{array}$ & 172 eventos & Varias regiones & $\begin{array}{l}1981- \\
1994\end{array}$ & Positivo & $\begin{array}{l}\text { Hulburt } \\
(2011)\end{array}$ \\
\hline $\begin{array}{l}\text { Fusiones y } \\
\text { adquisiciones }\end{array}$ & $\begin{array}{l}93 \text { estudios } \\
\text { empíricos }\end{array}$ & Varias regiones & $\begin{array}{l}1921- \\
2002\end{array}$ & Negativo & $\begin{array}{l}\text { King, } \\
\text { Dalton, } \\
\text { Daily, \& } \\
\text { Covin } \\
(2004)\end{array}$ \\
\hline $\begin{array}{c}\text { Fusiones y } \\
\text { adquisiciones }\end{array}$ & 69 empresas & Japón & $\begin{array}{l}1969- \\
1999\end{array}$ & Positivo & $\begin{array}{c}\text { Kruse, } \\
\text { Park, Park, } \\
\text { \& Suzuki } \\
(2007)\end{array}$ \\
\hline $\begin{array}{l}\text { Venta de } \\
\text { activos }\end{array}$ & 655 eventos & Reino Unido & $\begin{array}{l}1993- \\
1997\end{array}$ & Negativo & $\begin{array}{l}\text { Lee } \& \text { Lin } \\
\quad(2008)\end{array}$ \\
\hline $\begin{array}{c}\text { Fusiones y } \\
\text { adquisiciones }\end{array}$ & 118 empresas & India & $\begin{array}{l}1991- \\
2003\end{array}$ & Negativo & $\begin{array}{c}\text { Mantravadi } \\
\text { \& Reddy } \\
\text { (2008) }\end{array}$ \\
\hline $\begin{array}{c}\text { Subsidiaria y } \\
\text { empresa filial } \\
\text { parcial }\end{array}$ & $\begin{array}{c}91 \text { eventos ( } 30 \\
\text { subsidiarias } \\
\text { y } 61 \text { empresa } \\
\text { filial parcial) }\end{array}$ & Varias regiones & $\begin{array}{l}1981- \\
1988\end{array}$ & Positivo & $\begin{array}{l}\text { Michaely } \\
\text { \& Shaw } \\
(1995)\end{array}$ \\
\hline $\begin{array}{c}\text { Venta de } \\
\text { activos, } \\
\text { subsidiaria y } \\
\text { empresa filial } \\
\text { parcial }\end{array}$ & $\begin{array}{l}139 \text { ventas de } \\
\text { activos, } 106 \\
\text { subsidiarias y } \\
125 \text { empresas } \\
\text { filiales parciales }\end{array}$ & Varias regiones & $\begin{array}{c}1990- \\
1999\end{array}$ & Positivo & $\begin{array}{c}\text { Mulherin } \\
\text { \& Boone } \\
(2000)\end{array}$ \\
\hline $\begin{array}{c}\text { Empresa filial } \\
\text { parcial }\end{array}$ & 181 empresas & Varias regiones & $\begin{array}{l}1981- \\
1996\end{array}$ & Positivo & $\begin{array}{l}\text { Powers } \\
(2003)\end{array}$ \\
\hline
\end{tabular}




\begin{tabular}{|c|c|c|c|c|c|}
\hline $\begin{array}{c}\text { Fusiones y } \\
\text { adquisiciones }\end{array}$ & 61 eventos & Reino Unido & $\begin{array}{c}1988- \\
1990\end{array}$ & $\begin{array}{c}\text { Neutral: } \\
\text { depende } \\
\text { de las } \\
\text { circunstancias }\end{array}$ & $\begin{array}{c}\text { Schoenberg } \\
(2006)\end{array}$ \\
\hline Empresa filial & 93 eventos & Varias regiones & $\begin{array}{c}1963- \\
1981\end{array}$ & Positivo & $\begin{array}{c}\text { Schipper } \\
\& \text { Smith } \\
(1983)\end{array}$ \\
\hline $\begin{array}{c}\text { Subsidiaria y } \\
\text { empresa filial } \\
\text { parcial }\end{array}$ & 266 eventos & Taiwán & $\begin{array}{c}1995- \\
2004\end{array}$ & Positivo & Sun (2012) \\
\hline $\begin{array}{c}\text { Fusiones y } \\
\text { adquisiciones }\end{array}$ & 86 empresas & Japón & $1970-$ & Negativo & $\begin{array}{c}\text { Tsung- } \\
\text { ming \& } \\
\text { Hoshino } \\
(2002)\end{array}$ \\
\hline $\begin{array}{c}\text { Fusiones y } \\
\text { adquisiciones }\end{array}$ & 75 eventos & Varias regiones & $\begin{array}{c}1999- \\
1993\end{array}$ & Positivo & $\begin{array}{c}\text { Yook } \\
(2004)\end{array}$ \\
\hline Empresa filial & 36 eventos & Malasia & $1980-$ & Positivo & $\begin{array}{c}\text { Zakaria } \\
\text { ( Arnold } \\
(2016)\end{array}$ \\
\hline
\end{tabular}

Fuente: Elaboración propia.

Al observar la tabla anterior se pueden recalcar diversas situaciones específicas. En primer lugar, de acuerdo a los artículos analizados de la tabla 3, se muestra que el $67 \%$ de los mecanismos tienen efectos positivos en los resultados de las empresas. Esto quiere decir que sí ha funcionado su implementación puesto que se ha creado valor para los accionistas junto a un mejor rendimiento económico. En segundo lugar, se observa que para la RCN el mecanismo más propicio a ser utilizado es el de las desinversiones (comprendido por ventas de activos, empresa filial y empresa filial parcial) ya que en la mayoría de estudios presenta un efecto positivo en los resultados de la empresa. Por último, se distingue que el mecanismo de F\&A tiene un efecto, en mayor parte, negativo dentro de los estudios analizados por motivos explicados en el apartado 4. Cabe resaltar la poca intervención de países con economías emergentes para el estudio de la RCN ya que la mayor parte de las investigaciones en la tabla 3 han sido desarrolladas en Estados Unidos, (país pionero en el estudio de esta estrategia) o en la región europea con empresas, en su mayoría, de Reino Unido (Sánchez-Riofrío, Guerras-Martín, \& Forcadell, 2016). 


\section{Conclusiones}

El objetivo de la presente investigación fue analizar conceptualmente el estudio de la RCN y su efecto en los resultados de las empresas. De acuerdo a la evidencia presentada, en primer lugar encontramos que la RCN se divide en tres direcciones de desarrollo: (i) la reorientación corporativa que implica desinversiones de negocios periféricos y especialización en las actividades clave, (ii) el reposicionamiento corporativo que redefine la actividad principal de la empresa hacia un nuevo sector mediante desinversiones y adquisiciones, y (iii) la liquidación de activos que consiste en la terminación de las operaciones del negocio junto al inicio de un proceso de desinversiones encaminado a pagar la totalidad de los pasivos.

En segundo lugar, existen dos mecanismos para implementar los cambios en la RCN que son: (i) las desinversiones, y (ii) las fusiones y adquisiciones. Entre ambos, el mecanismo más habitual según los estudios empíricos son las desinversiones puesto que implican acciones frecuentemente utilizadas como transferir activos, líneas de negocios, o inversiones mediante su venta a otras empresas.

En tercer lugar, se mostró el efecto obtenido por la RCN en las empresas de acuerdo a distintos estudios empíricos. La literatura académica indica que por lo general los diversos mecanismos tienen efectos positivos en los resultados de las empresas. Sin embargo, de acuerdo a varios autores las F\&A son más propensas a destruir, en vez de crear valor por lo cual no poseen realmente el efecto positivo deseado por los administradores.

En cuarto lugar, se observa una gran escasez de artículos científicos que traten acerca de la RCN en empresas latinoamericanas. Los artículos analizados en bases de datos como WoS, Scopus, Dialnet, Redalyc, entre otros, no muestran artículos sobre esta temática específica lo cual comprendería una nueva línea de investigación para Latinoamérica.

Como toda investigación, el presente trabajo tiene sus limitaciones. Primero, se analizó un número limitado de artículos científicos relacionados al tema. Segundo, la RCN se ha examinado, en su mayoría, bajo tres enfoques por la literatura. Sin embargo, en este trabajo solo se tomó en consideración al EBR de Penrose (1959) mas no la teoría de la agencia (Jensen, 1986) ni los costos 
de transacción (Williamson, 1975). Finalmente, el efecto tanto positivo como negativo que obtienen las empresas por la RCN fue mostrado de acuerdo a los trabajos de diversos autores y no mediante a la elaboración de un estudio empírico propio para cada uno de los mecanismos propuestos debido a lo extenso y complejo de su análisis.

BiBLIOGRAFÍA

ABDUL-MAGID, G., \& HARDY, T. (2004). "Do asset sales lead to improvements in operating performance?" Applied Economics, 36(8), 865-871.

AGRAWAL, A., JAFFE, J., \& MANDELKER, G. (1992). “The PostMerger Performance of Acquiring Firms: A Re-Examination of an Anomaly". The Journal of Finance, 47(4), 1605-1621. ALLEN, J., LUMMER, S., MCCONELL, J., \& REED, D. (1995).

"Can Takeover Losses Explain Spin-Off Gains?" Journal of Financial and Quantitative Analysis, 30(4), 465-485.

América Economía. (5 de Enero de 2017). Petrobras completó venta de activos en Chile por US\$470 millones. Recuperado el 25 de Enero de 2017, de http://www.americaeconomia. $\mathrm{com} /$ negocios-industrias/fusiones-adquisiciones/petrobrascompleto-venta-de-activos-en-chile-por-us470

BENA, J., \& LI, K. (2012). "Corporate Innovations and Mergers and Acquisitions". The Journal of Finance, 69(5), 1-64.

BERGH, D., \& LIM, E. (2008). "Learning how to Restructure: Absorptive Capacityand Improvisational Views of Restructuring Actions and Performance". Strategic Management Journal, 29(6), 593-616.

BERGH, D., JOHNSON, R., \& DEWITT, R. (2008). "Restructuring through spin-off or sell-off: Transforming information asymmetries into financial gain”. Strategic Management Journal, 29(2), 133-148.

BHANA, N. (2004). "Performance of corporate restructurings through spin-offs: Evidence from JSE-listed companies". Investment Analysts Journal(60), 5-15. 
BHANA, N. (2006). "The effect of corporate divestments on shareholder wealth: The South African experience". Investment Analysts Journal(63), 19-30.

BLANCO-CALLEJO, M., FORCADELL, F., \& GUERRASMARTÍN, L. (2009). "La estrategia de reestructuración de la cartera de negocios orientada a la creación de valor". Revista europea de dirección y economía de la empresa, 5(2), 29-48.

BOONE, A., HAUSHALTER, D., \& MIKKELSON, W. (2003). "An Investigation of the Gains from Specialized Equity Claims". Financial Management, 32(3), 67-83.

BORISOVA, G., JOHN, K., \& SALOTTI, V. (2013). “The value of financing through cross-border asset sales: Shareholder returns and liquidity”. Journal of Corporate Finance, 22, 320-344.

BOWMAN, E., SINGH, H., USEEM, M., \& BHADURY, R. (1999). "When does restructuring improve economic performance”. California Management Review, 41(2), 33-54. BRIS, A., BRISLEY, N., \& CABOLIS, C. (2008). "Adopting better corporate governance: Evidence from cross-border mergers". Journal of Corporate Finance, 14(3), 224-240.

BYERLY, R., LAMONTB, B., \& KEASLER, T. (2003). "Business Portfolio Restructuring, PriorDiversification Posture and Investor Reactions". Managerial and Decision Economics, 24(8), 535-548.

CHENG, P., AERTS, W., \& JORISSEN, A. (2010). "Earnings Management, Asset Restructuring, and the Threat of Exchange Delisting in an Earnings-based Regulatory Regime". Corporate Governance: An International Review, 18(5), 438-456.

CHOE, S., \& ROEHL, T. (2007). "What to shed and what to keep: Corporate transformation in Korean Business Groups". Long Range Planning, 40(4-5), 465-487.

COLAK, G. (2010). "Diversification, Refocusing and Firm Value". European Financial Management, 16(3), 422 - 448.

DALEY, L., MEHROTRA, V., \& SIVAKUMAR, R. (1997). "Corporate focus and value cration: Evidence from spinoffs". Journal of Financial Economics, 45(2), 257-281. 
DAMARAJU, N., BARNEY, J., \& MAKHIJA, A. (2011). "Real Options in Divestment Alternatives". Strategic Management Journal, 36(5), 1-38.

DAVID, F. (2013). "Administración Estratégica". México D.F.: Pearson.

DESAI, C., KLOCK, M., \& MANSI, S. (2011). "On the Acquisition of Equity Carve-Outs". Journal of Banking and Finance, 35(12), 3432-2449.

DESAI, H., \& JAIN, P. C. (1999). "Firm Performance and Focus: Long-Run Stock Market Performance Following Spinoffs". Journal of Financial Economics, 54(1), 75-101.

DESS, G., LUMPKIN, T., \& EISNER, A. (2011). “Administración estratégica: Textos y casos”. México D.F: McGraw-Hill.

DUHAIME, I., \& GRANT, J. (1984). "Factors Influencing Divestment Decision Making: Evidence from a Field Study". Strategic Management Journal, 5(4), 301-318. El Economista. (08 de Enero de 2018). Economía/Empresas.- OHL aprueba en junta la venta de concesiones y un 'bonus' de 18 millones para su consejero delegado. Obtenido de http://www.eleconomista.es/ mercados-cotizaciones/noticias/8853302/01/18/EconomiaEmpresas-OHL-aprueba-en-junta-la-venta-de-concesionesy-un-bonus-de-18-millones-para-su-consejero-delegado. html El País. (11 de Agosto de 2015). De una sola empresa a siete distintas: así pasa Google a ser Alphabet. Recuperado el 18 de Enero de 2017, de http://tecnologia.elpais.com/ tecnologia/2015/08/11/actualidad/1439276220_801037. html

FERNÁNDEZ,J., GARAY,U., \&PABLO,E.(2010). “Determinants of shareholders' returns following announcements of asset sales: Evidence from Latin America”. Revista Latinoamericana de Administración(45), 40-58.

GALLARDO, J. (2012). “Administración Estratégica: De la visión a la ejecución”. México D.F.: Alfaomega.

GIBBS, P. (1993). "Determinants of corporate restructuring: The relative importance of corporate governance, takeover threat, 
and free cash flow”. Strategic Management Journal, 14(1), 5168.

HAYNES, M., THOMPSON, S., \& WRIGHT, M. (2002). "The Impact of Divestment on Firm Performance: Empirical Evidence from a Panel of UK Companies". Journal of Industrial Economics, 50(2), 173-196.

HILL, C., \& JONES, G. (2009). “Administración Estratégica”. México D.F.: McGraw-Hill.

HILLIER, D., MCCOLGAN, P., \& WEREMA, S. (2009). "Asset Sales and Firm Strategy: An Analysis of Divestitures by UK Companies". European Journal of Finance, 15(1), 71-87.

HITE, G., \& OWERS, J. (1983). "Security price reactions around corporate spin-off announcements". Journal of Financial Economics, 12(4), 409-436.

HITT, M., IRELAND, D., \& HOSKISSON, R. (2015). "Administración Estratégica". México D.F.: Cengage Learning Editores S.A.

HOMBERG, F., ROST, K., \& OSTERLOH, M. (2007). "Do synergies exist in related acquisitions? - A meta-analysis of acquisition studies". Review of Managerial Science, 3(2), 75116.

HOSKISSON, R., CANNELLA, A., TIHANYI, L., \& FARACI, R. (2004). "Asset Restructuring and Business Group Affiliation in French Civil Law Countries". Strategic Management Journal, 25(6), 525-539.

HOSKISSON, R., JOHNSON, R., TIHANYI, L., \& WHITE, R. (2005). "Diversified Business Groups and Corporate Refocusing in Emerging Economies”. Journal of Management, 31(6), 941-965.

HULBURT, H. (2011). "Equity Carve-Outs And Changes In Corporate Control”. The Journal of Applied Business Research, 19(1), 29-41.

INGA-HENRÍQUEZ， R., \& CABA-GAJARDO, S. (2015). "El fracaso de Blockbuster y el éxito de Netflix, lecciones aprendidas y otras por aprender”. Revista Gestión de las 
Personas y Tecnología, 8(23), 39-48.

ISMAIL, T., ABDOU, A., \& ANNIS, R. (2011). "Review of Literature Linking Corporate Performance to Mergers and Acquisitions". The Review of Financial and Accounting Studies(1), 89-104.

JENSEN, M. (1986). "Agency Cost of Free Cash Flow, Corporate Finance". American Economic Review, 76(2), 323-329.

JOHN, K., \& OFEK, E. (1995). "Asset sales and increase in focus". Journal of Financial Economics, 37(1), 105-126.

KIM, E., \& SCHATZBERG, J. (1987). "Voluntary corporate liquidations". Journal of Financial Economics, 311-328.

KING, D., DALTON, D., DAILY, C., \& COVIN, J. (2004). "Meta-analyses of Post Acquisition Performance: Indications of Unidentified Moderators". Strategic Management Journal, 25(2), 187-200.

KRUSE, T., PARK, H., PARK, K., \& SUZUKI, K. (2007). "Longterm performance following mergers of Japanese companies: The effect of diversification and affiliation". Pacific-Basin Finance Journal, 15(2), 154-172.

LANG, L., POULSEN, A., \& STULZ, R. (1995). "Asset sales, firm performance, and the agency costs of managerial discretion". Journal of Financial Economics, 37(1), 3-37.

LEE, E., \& LIN, S. (2008). "Corporate Sell-Offs in the UK: Use of Proceeds, Financial Distress and Long-Run Impact on Shareholder Wealth". European Financial Management, 14(2), 222-242.

LOCKETT, A., \& THOMPSON, S. (2001). "The resource-based view and economics". Journal of Management, 27(6), 723754.

MALIK, M., ANUAR, M., KHAN, S., \& KHAN, F. (2014). "Mergers and Acquisitions: A Conceptual Review". International Journal of Accounting and Financial Reporting, 4(2), 2162-3082.

MANTRAVADI, P., \& REDDY, V. (2008). "Post-Merger Performance of Acquiring Firms from Different Industries in India”. International Research Journal of Finance and Economics(22), 192-204. 
MARKIDES, C. (1992). "Consecuences of Corporate Refocusing: Ex Ante Evidence". The Academy of Management Journal, 35(2), 398-412.

McKinsey \& Company. (2001). Do carve-outs make sense? Recuperado el 18 de Enero de 2017, de http://www.mckinsey. $\mathrm{com} /$ business-functions/strategy-and-corporate-finance/ourinsights/do-carve-outs-make-sense

MICHAELY, R., \& SHAW, W. (1995). "The Choice of Going Public: Spin-offs vs. Carve-outs". Financial Management, 24(3), 5-21.

MOLINA-YCAZA, D., \& SÁNCHEZ-RIOFRÍO, A. (2016). "Factores de competitividad orientados a la pequeńa y mediana empresa (PYME) en América Latina: Revisión de la literatura”. Revista San Gregorio(15), 104-111.

MULHERIN, H., \& BOONE, A. (2000). "Comparing acquisitions and divestitures". Journal of Corporate Finance, 6(2), 117139.

OFEK, E. (1993). "Capital structure and firm response to poor performance". Journal of Financial Economics, 34(1), 3-30.

PENROSE, E. (1959). "The theory of the growth of the firm". Nueva York: NY: Wiley.

PEROTTI, E., \& ROSSETTO, S. (2007). "Unlocking value: Equity carve outs as strategic real options". Journal of Corporate Finance, 13(5), 771-792.

POWERS, E. (2003). "Deciphering the Motives for Equity CarveOuts". Journal of Financial Research, 26(1), 31-50.

RENNEBOOG, L., \& SZILAGYI, P. (2006). "Corporate Restructuring and Bondholder Wealth". European Financial Management, 14(4), 1-27.

ROBBINS, D., \& PEARCE, J. (1992). "Turnaround: Retrenchment and recovery". Strategic Management Journal, 13, 287-300. Samsung. (2005). Recuperado el 18 de Enero de 2017, de http://www.samsung.com/es/aboutsamsung/samsung/ history_08/

SÁNCHEZ-RIOFRÍO， A., GUERRAS-MARTÍN, L., \& FORCADELL, F. (2015). "Business portfolio restructuring: a comprehensive bibliometric review". Scientometrics, 102(3), 
1921-1950.

SÁNCHEZ-RIOFRÍO, A., GUERRAS-MARTÍN, L., \& FORCADELL, F. (2016). "Reestructuración de la Cartera de Negocios: Revisión de la Literatura”. PODIUM(30), 71-83.

SÁNCHEZ-RIOFRÍO, A., GUERRAS-MARTÍN, L., \& FORCADELL, F. (2017). "Who's who in business portfolio restructuring research: The intellectual structure”. Revista española de Documentación Científica, 40(1), 1-15.

SCHIPPER, K., \& SMITH, A. (1983). "Effects of recontracting on shareholder wealth: The case of voluntary spin-offs". Journal of Financial Economics, 12(4), 437-467.

SCHIPPER, K., \& SMITH, A. (1986). "A Comparison of Equity Carve-Outs and Seasoned Equity Offerings". Journal of Financiat Economics, 15(1-2), 153-186.

SCHOENBERG, R. (2006). "Measuring the Performance of Corporate Acquisitions: An Empirical Comparison of Alternative Metrics”. British Journal of Management, 17(4), 361-370.

SCHONHAAR, S., NIPPA, M., \& PIDUN, U. (2014). "From patchwork to theory development: Mapping and advancing research about business portfolio restructuring". Management Review Quarterly, 64(3), 157-200.

SEWARD, J., \& WALSH, J. (1996). "The governance and control of voluntary corporate spin-offs". Strategic Management Journal, 17(1), 25-39.

SHLEIFER, A., \& VISHNY, R. (1992). "Liquidation Values and Debt Capacity: A Market Equilibrium Approach". The Journal of Finance, 47(4), 1343-1366.

SIBILKOV, V., STRASKA, M., \& WALLER, G. (2013). “The value implications of restrictions on asset sales". Review of Financial Economics, 22(3), 98-108.

SLOVIN, M., SUSHKA, M., \& FERRARO, S. (1995). "A comparison of the information conveyed by equity carve-outs, spin-offs, and asset sell-offs". Journal of Financial Economics, 37(1), 89-104.

SULAIMAN, L. (2012). "Does Restructuring Improve Performance? An Industry Analysis Of Nigerian Oil \& Gas Sector”. Research 
Journal of Finance and Accounting, 3(6), 55-62.

SUN, M. (2012). "Impact of Divestiture Activities on Corporate Performance: Evidence from Listed Firms in Taiwan". The International Journal of Business and Finance Research, 6(2), 59-67.

TARJIZÁN, J. (2008). "Fundamentos de estrategia empresarial". México D.F.: Alfaomega. The Wall Street Journal. (16 de Septiembre de 2015). SABMiller recibe interés de compra de AB InBev. Recuperado el 18 de Enero de 2017, de http://lat. wsj.com/articles/SB12177959380318133490704581236750 665080640

THOMPSON, A., STRICKLAND, A., \& GAMBLE, J. (2008). "Administración Estratégica". México D.F.: McGraw-Hill. THOMPSON, A., STRICKLAND, A., \& GAMBLE, J. (2012). "Administración Estratégica: teoría y casos". México D.F.: McGraw-Hill.

TSUNG-MING, Y., \& HOSHINO, Y. (2002). "Productivity and Operating Performance of Japanese Merging Firms: Keiretsurelated and Independent Mergers". Japan and the World Economy, 14(3), 347-366.

UDDIN, H. (2010). "Corporate spin-offs and shareholders' value: Evidence from Singapore". The International Journal of Business and Finance Research, 4(4), 43-57.

WALLIN, M. (2012). "The Bibliometric Structure of Spin-Off Literature". Innovation: Management, Policy \& Practice, 14(2), 2056-2095.

WILLIAMSON, O. (1975). "Markets and hierarchies: Analysis and antitrust implications". Nueva York: NY: Free Press.

WOOD, S. (2001). "Regulatory constrained portfolio restructuring: The US department store industry in the 1990s". Environment and Planning A, 33(7), 1279-1304.

YOOK, K. (2004). "The Measurement of Post-Acquisition Performance Using EVA". Journal of Business \& Economics, 43(3-4), 67-84.

ZAKARIA, N., \& ARNOLD, G. (2016). "Spin-Off and Value Creation: The Case of Malaysia". International Review of Business Research Papers, 12(1), 121-143. 
Fecha de Recepción del Artículo: 10 de enero de 2017 Fecha de Aceptación: 30 de marzo de 2017 